\title{
Imaging of Nonprostate Cancers Using PSMA-Targeted Radiotracers: Rationale, Current State of the Field, and a Call to Arms
}

\author{
Roberto A. Salas Fragomeni ${ }^{1}$, Tali Amir ${ }^{1}$, Sara Sheikhbahaei ${ }^{1}$, Susan C. Harvey ${ }^{1}$, Mehrbod S. Javadi ${ }^{1}$, Lilja B. Solnes ${ }^{1}$, \\ Ana P. Kiess ${ }^{2}$, Mohamad E. Allaf ${ }^{3}$, Martin G. Pomper ${ }^{1,3}$, Michael A. Gorin ${ }^{1,3}$, and Steven P. Rowe ${ }^{1,3}$ \\ ${ }^{1}$ Russell H. Morgan Department of Radiology and Radiological Science, Johns Hopkins University School of Medicine, Baltimore, \\ Maryland; ${ }^{2}$ Department of Radiation Oncology and Molecular Radiation Sciences, Johns Hopkins University School of Medicine, \\ Baltimore, Maryland; and ${ }^{3}$ James Buchanan Brady Urological Institute and Department of Urology, Johns Hopkins University School \\ of Medicine, Baltimore, Maryland
}

\begin{abstract}
Prostate-specific membrane antigen (PSMA) is a type II transmembrane glycoprotein that is highly overexpressed on prostate cancer epithelial cells and for which there is a growing body of literature examining the role of small-molecule and antibody radiotracers targeted against this protein for prostate cancer detection and therapy. Despite its name, PSMA is also expressed, to varying degrees, in the neovasculature of a wide variety of nonprostate cancers; indeed, the pathology literature is replete with promising immunohistochemistry findings. Several groups have begun to correlate those pathologylevel results with in vivo imaging and therapy in nonprostate cancers using the same PSMA-targeted agents that have been so successful in prostate cancer. The potential to leverage radiotracers targeted to PSMA beyond prostate cancer is a promising approach for many cancers, and PSMA-targeted agents may be able to supplement or fill gaps left by other agents. However, to date, most of the reported findings with PSMA-targeted radiotracers in nonprostate malignancies have been in case reports and small case series, and the field must adopt a more thorough approach to the design and execution of larger prospective trials to realize the potential of these promising agents outside prostate cancer.
\end{abstract}

Key Words: PSMA; PET; breast cancer; lung cancer; renal cell carcinoma

J Nucl Med 2018; 59:871-877

DOI: 10.2967/jnumed.117.203570

$\mathbf{P}$ rostate-specific membrane antigen (PSMA) is a type II transmembrane glycoprotein initially recognized by the monoclonal antibody 7EII-C5.3 in LNCaP cell line extracts $(1,2)$. PSMA exhibits in vitro neuropeptidase activity (3), as well as folate hydrolase activity (4). On the basis of these enzymatic characteristics, the nomenclature committee of the International Union of Biochemistry and Molecular Biology has recommended that PSMA carry the formal name of glutamate carboxypeptidase (5) (enzyme EC 3.4.17.21); this decision is perhaps not surprising given that

Received Dec. 19, 2017; revision accepted Feb. 23, 2018.

For correspondence or reprints contact: Steven P. Rowe, Johns Hopkins School of Medicine, 600 N. Wolfe St., Baltimore, MD 21287.

E-mail: srowe8@jhmi.edu

Published online Mar. 15, 2018.

COPYRIGHT (C 2018 by the Society of Nuclear Medicine and Molecular Imaging. this enzyme is expressed in a wide variety of normal and pathologic tissues outside the prostate.

Despite being a misnomer, PSMA has become the preferred term within the imaging community, and with hundreds of publications on PSMA-targeted PET now available, it is likely to remain so. The high expression of PSMA in prostate epithelium $(1,2)$ has enabled PSMA-targeted agents to demonstrate important therapeutic (6) and diagnostic (7) potential for prostate cancer in a variety of clinical scenarios from initial staging to biochemical recurrence and widespread metastatic disease.

A lower, yet still significant, amount of PSMA expression has been found in the normal salivary glands, proximal renal tubules, brain, and small intestine by immunohistochemistry $(8-11)$ and messenger RNA (12), and these findings also manifest during patient imaging (13). Furthermore, PSMA expression has been shown in the neovasculature of a wide array of nonprostate malignancies, raising the possibility of PSMA theranostic applications outside prostate cancer (Tables 1 and 2). Herein, we review the available pathology and imaging literature and outline such potential applications.

\section{LITERATURE REVIEW}

A literature review of PubMed/Medline was performed on December 6, 2017, using the following search terms: "glutamate carboxypeptidase II," "prostate specific membrane antigen," "PSMA," "PET," and "positron emission tomography." No date limit or language restrictions were applied. The goal was to identify relevant published studies reporting PSMA expression in tumor cells, patient samples, or in vivo imaging. The inclusion process is summarized in Supplemental Figure 1. In total, 2,990 studies were identified. Studies pertaining solely to prostate cancer were excluded, leaving 150 studies for review. Of these, 15 were excluded because they did not focus on PSMA expression in the neoplastic setting. Only studies using surgical specimens or tumor extracts for immunohistochemistry, and clearly denoting the histopathology of the tumor, were included in the tables (Table 1) and used for pooled estimates. No antibody restriction was applied. For studies reporting different staining grading, any PSMA histologic staining, regardless of the strength, was considered to be positive for PSMA expression. The results were stratified according to the most common cancers in the United States (14), excluding prostate cancer. 
TABLE 1

PSMA Expression in Nonprostate Cancers

\begin{tabular}{|c|c|c|c|}
\hline Tissue & Tumor cells ${ }^{*}$ & Tumor vascular endothelium $(\%)$ & Imaging \\
\hline Breast adenocarcinoma & $1 / 107(1 \%)(2,8,16,18,21)$ & $74 / 99(75 \%)(2,9,16,18,54)$ & ${ }^{111}$ In J591 (23,24); ${ }^{68}$ Ga HBED-CC $(20,25)$ \\
\hline NSCLC & $66 / 420(16 \%)(2,15,16,30,31,54)$ & $222 / 374(59 \%)(8,9,30,31,33)$ & ${ }^{111}$ In-J591 (23); ${ }^{68} \mathrm{Ga}-H B E D-C C ~(32,33)$ \\
\hline $\begin{array}{l}\text { Colorectal } \\
\text { adenocarcinoma }\end{array}$ & $20 / 206(10 \%)(8,11,15,16,41)$ & $193 / 254(76 \%)(8,11,41,42)$ & ${ }^{111}$ In-J591 (23,24); ${ }^{68}$ Ga-HBED-CC $(43,45,46)$ \\
\hline $\begin{array}{l}\text { Transitional cell } \\
\text { carcinoma }\end{array}$ & $22 / 128(17 \%)(11,15,16,48,54)$ & $31 / 107(29 \%)(8,9,11,48)$ & ${ }^{111}$ In-J591 (23,24); ${ }^{68} \mathrm{Ga}-\mathrm{HBED}-\mathrm{CC}(49) ;{ }^{18} \mathrm{~F}-\mathrm{DCFPyL}(50)$ \\
\hline Glioblastoma multiforme & $3 / 58(5 \%)(8,15,21)$ & $40 / 40(100 \%)(8,21,67,68)$ & ${ }^{68} \mathrm{Ga}-\mathrm{HBED}-\mathrm{CC}(69,70) ;{ }^{18} \mathrm{~F}-\mathrm{DCFPyL}(68)$ \\
\hline $\begin{array}{l}\text { Pancreatic ductal } \\
\text { adenocarcinoma }\end{array}$ & $112 / 167(67 \%)(8,15,19)$ & $4 / 4(100 \%)(8)$ & ${ }^{111}$ In-J591 (23); ${ }^{68 G a-H B E D-C C ~(73) ~}$ \\
\hline Gastric adenocarcinoma & $15 / 202(7 \%)(15,16,19,41)$ & $90 / 119(76 \%)(41)$ & ${ }^{111}$ In-J591 (24) \\
\hline
\end{tabular}

\section{BREAST CANCER}

Breast cancer is currently the most common cancer in the United States, with an estimated 250,000 new cases in 2017 (14). Although breast tissue has shown weak to moderate PSMA expression in normal alveoli, globule cells, and terminal duct epithelia $(15,16)$, strong PSMA expression has been shown in primary and metastatic breast cancer neovasculature by polymerase chain reaction (17) and immunohistochemistry, with tumor neovasculature PSMA staining being present in $74 \%-100 \%$ of all samples $(8,18,19)$. A correlation of PSMA expression between metastatic sites and primary tumors has been demonstrated in vivo and in vitro $(18,20,21)$. Early human clinical studies demonstrated activity in breast tissue (22) and breast carcinoma using ${ }^{111}$ In-J591 (23) planar imaging, as well as ${ }^{68} \mathrm{Ga}$-PSMAHBED-CC ( $N, N^{\prime}$-bis[2-hydroxy-5-(carboxyethyl)benzyl]ethylenediamine- $N, N^{\prime}$-diacetic acid) PET/CT (Fig. 1) (20,23-26). For instance, Sathekge et al. demonstrated PSMA expression in $84 \%$ of 81 suspected metastatic lesions in 19 breast carcinoma patients who were undergoing PSMA-targeted PET imaging (20). However, benign breast conditions mimicking tumors have also been reported (27).

Increased PSMA expression in breast cancer has been associated with statistically lower overall survival, higher median tumor size, higher cell proliferation, and higher nuclear grades (18). PSMA expression was more likely to be higher in estrogen receptor- and progesterone receptor-negative tumors than in estrogen receptorand progesterone receptor-positive tumors (18). No correlation was identified between PSMA expression and the propensity for lymph node involvement (18). There was variability in HER2/neu expression relative to PSMA expression (18). Overall, these findings suggest that PSMA-targeted PET imaging can conceivably act as an imaging biomarker of tumor aggressiveness and may play a role in

\section{NOTEWORTHY}

- PSMA is expressed by a variety of nonprostate cancers, often on the endothelium of tumor-associated neovasculature.

- Among the most common nonprostate cancers to demonstrate PSMA expression are breast, lung, colorectal, and renal cell carcinoma.

- Prospective trials definitively evaluating PSMA-targeted agents for imaging and therapy in nonprostate cancers are needed. the noninvasive identification of aggressive breast cancers, thereby helping to appropriately risk-stratify patients.

The role of standard oncology PET imaging with ${ }^{18} \mathrm{~F}-\mathrm{FDG}$ in breast cancer remains somewhat controversial. The National Comprehensive Cancer Network guidelines encourage the use of ${ }^{18} \mathrm{~F}$ FDG PET in patients with initial disease of clinical stage T3N1 or higher and also recommend ${ }^{18} \mathrm{~F}$-FDG PET in patients with recurrent disease for restaging (28). Imaging with ${ }^{18} \mathrm{~F}-\mathrm{FDG}$ PET is generally not recommended for clinically localized $\mathrm{T} 1$ or $\mathrm{T} 2$ tumors or for locally advanced T2N1 or T3N0 disease. Although it is unlikely that PSMA-targeted PET will contribute any information to standard imaging in patients with $\mathrm{T} 1$ or $\mathrm{T} 2$ tumors and no evidence of locoregional lymph node involvement, the high tumorto-background ratio and high-contrast resolution of PSMA-targeted radiotracers may prove useful in ruling out occult metastases in locally advanced breast cancer. The improved specificity and imaging characteristics of PSMA-targeted agents relative to ${ }^{18} \mathrm{~F}-\mathrm{FDG}$ may allow us, over the long term, to successfully implement PET in more breast cancer patients than current guidelines recommend.

Further, the expression of PSMA on lobular breast cancers suggests a role for PSMA-based imaging agents in evaluating lobular tumors $(18,20)$. Currently, ${ }^{18} \mathrm{~F}$-FDG PET often suffers from low radiotracer uptake in lobular breast cancer, limiting evaluation of the extent of primary tumors and lowering sensitivity for metastatic disease. Indeed, it is this potential application of PSMA-targeted imaging in breast cancer that should perhaps be most urgently explored, as the need for reliable agents for systemic staging in lobular breast cancer has been long-standing.

Furthermore, the rise of radiopharmaceutical therapy with PSMAtargeted agents (29) may also open new methods to treat patients with progressive, advanced disease when other systemic therapeutic options have failed (6). Breast cancer patients who have failed multiple lines of therapy but whose PSMA-targeted PET imaging demonstrates highly avid radiotracer uptake may be considered for palliative therapy with PSMA-targeted therapeutics. Ultimately, larger, preferably prospective, studies of PSMA-targeted PET in patients with breast cancer will help to more clearly define the role of these imaging agents in addressing important clinical questions and in favorably altering patient management.

\section{LUNG CANCER}

Lung cancer is the second most common type of cancer in the United States, with over 220,000 new cases a year (14). Although 
TABLE 2

PSMA Expression in Renal Tumors

\begin{tabular}{lccc}
\hline \multicolumn{1}{c}{ Tissue } & Tumor cells ${ }^{*}(\%)$ & Tumor vascular endothelium ${ }^{*}(\%)$ & Imaging \\
\hline Clear cell RCC & $5 / 349(1 \%)(8,15,16,51-54)$ & $259 / 310(84 \%)(8,51-54)$ & $\begin{array}{c}{ }^{111} \text { In-7E11-C5.3 (61); }{ }^{68} \text { Ga-HBED-CC } \\
(56,59,60,62,63,83) ;{ }^{18} \mathrm{~F}-\mathrm{DCFPyL}(57,65,84)\end{array}$ \\
\hline Papillary RCC & $5 / 109(5 \%)(15,16,51-53)$ & $16 / 57(28 \%)(51-53)$ & ${ }^{68}$ Ga-HBED-CC $(59,63)$ \\
Chromophobe RCC & $0 / 57(0 \%)(15,16,51-53)$ & $23 / 38(61 \%)(51-53)$ & ${ }^{68}$ Ga-HBED-CC $(59,60)$ \\
Oncocytoma & $0 / 47(0 \%)(16,51-53)$ & $26 / 45(58 \%)(51-53)$ & \\
Angiomyolipoma & $0 / 21(0 \%)(8,52)$ & $4 / 20(20 \%)(8,52)$ & \\
\hline
\end{tabular}

*Data are percentage of samples with stained cells or vasculature in relation to total number of pooled samples.

no PSMA expression has been seen in normal lung tissue $(2,8,15,16)$, tumor epithelial cells from various histologic types of lung cancer, as well as neovascular endothelial cells, have demonstrated PSMA expression $(8,16,19,30)$. Furthermore, PSMA expression has been associated with higher histologic grades in non-small cell lung cancer (NSCLC) (31).

Although PSMA expression in NSCLC cells is detected in approximately $16 \%$ of tumor samples, tumor neovasculature PSMA expression is seen in approximately 59\% of NSCLC tumor samples and varies according to tumor histology: neovasculature expression in squamous cell carcinoma and large cell carcinoma was $64 \%$ and $71 \%$, respectively, whereas PSMA expression in lung adenocarcinoma was lower, seen in approximately $45 \%$ of samples $(30,31)$. In small cell lung cancer (SCLC), no PSMA expression was observed in tumor cells, whereas tumor neovasculature expressed PSMA in approximately $70 \%$ of cases (30). The lack of PSMA expression in normal lung tissue, combined with generally positive expression in neovasculature and tumor cells, favors the potential for highcontrast images and desirable tumor-to-background ratios in PSMAtargeted PET imaging of lung neoplasms.

Furthermore, in vivo expression of PSMA in NSCLC has been demonstrated in primary tumors, lymph nodes, and bone metastasis with ${ }^{111} \mathrm{In}-\mathrm{J} 591$ (23) planar imaging, as well as with ${ }^{68} \mathrm{Ga}-$ PSMA PET/CT $(32,33)$. In one important study, the $\mathrm{SUV}_{\max }$ for NSCLC lesions was so high that they could not be reliably distinguished from prostate cancer lesions metastatic to the lung (33).

${ }^{18}$ F-FDG PET/CT plays an important role in suspected or proven lung cancer cases through characterization of pulmonary nodules, initial staging, and subsequent restaging (34). An important limitation of ${ }^{18} \mathrm{~F}$-FDG PET/CT is its lack of specificity and the potential overlap of infections, inflammatory lesions, and radiation and
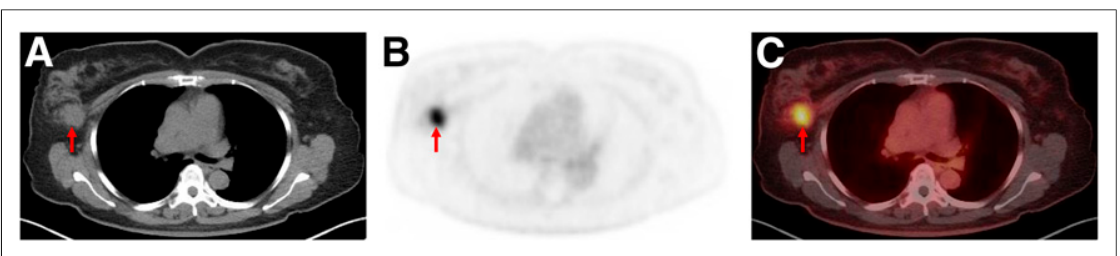

FIGURE 1. Axial unenhanced attenuation-corrected CT (A), axial ${ }^{18} \mathrm{~F}-\mathrm{DCFPyL} P E T(\mathrm{~B})$, and axial ${ }^{18}$ F-DCFPyL PET/CT (C) images from 65-y-old woman with right-sided, triple-negative, biopsyproven breast cancer showing intense radiotracer uptake in primary tumor (arrows). No evidence of local lymph node involvement or metastatic disease was seen on PET scan, and lymph node sampling at time of surgery was negative for disease involvement. surgical changes with metabolically active tumor, as well as potential sensitivity limitations in the evaluation of relatively metabolically inactive lesions, including adenocarcinoma in situ and carcinoid tumors (35).

These factors could potentially limit the utility of PSMAtargeted imaging and thus far have not been completely evaluated. For instance, although infections such as tuberculosis $(33,36)$, as well as chronically inflamed tissues (37), can show PSMA-targeted radiotracer uptake, the potential for higher specificity than is achievable with ${ }^{18} \mathrm{~F}$-FDG could indicate a promising role for PSMA-targeted agents in NSCLC and SCLC in terms of differentiating reactive mediastinal and hilar lymph nodes and inflammatory lung parenchymal findings from true tumor involvement.

One third of patients with NSCLC present with locally advanced disease with an estimated progression-free survival of $8 \mathrm{mo}$ and an approximate 5-y survival of $15 \%(38,39)$. Recent advances in immunotherapy have shown promising improvement in progressionfree survival, with durable results in responders, which represent approximately $25 \%$ of treated patients (40).

The utility of PSMA-targeted imaging in immunotherapy response is yet to be determined. However, for nonresponders, PSMAtargeted imaging may play a role in neovasculature-related therapies. Given the high expression of PSMA in tumor neovasculature in many lung cancers, PSMA-targeted imaging may act as an in vivo readout of neovascular density. The use of neovasculature-targeting therapeutic agents such as bevacizumab and tyrosine kinase inhibitors in lung cancer then suggests that PSMA-targeted imaging may predict response to such agents and also provide a means to track early evidence of response to therapy.

For patients who have limited therapy options, have failed multiple lines of therapy, or have rapidly progressive SCLC, it is possible that PSMA-targeted radiopharmaceutical therapy may provide a novel treatment modality. Prospective trials of NSCLC and SCLC patients are needed to investigate the diagnostic accuracy of PSMA-targeted imaging agents for metastatic disease, predict response to neovasculature-targeted therapies, and select patients for PSMA-targeted radiopharmaceutical therapy.

\section{COLORECTAL CANCER}

Colorectal cancer is the fourth most common type of cancer in the United States, with 135,000 new cases each year (14). Expression 
of PSMA has been demonstrated in colonic mucosa (specifically, chromogranin-positive cells of neuroendocrine origin in the deep aspects of the colonic crypts $(11,15))$. Although primary colorectal adenocarcinoma tumor cells infrequently show immunoreactivity, tumor neovasculature demonstrates increased PSMA expression $(2,8,11,16,41)$, as do lymph nodes containing metastatic disease (41). Higher-grade tumors tend to have higher PSMA expression (41) and a higher likelihood of distant metastases and vascular invasion (42). However, no statistical difference was seen in overall survival or disease-free survival based on expression levels of PSMA (41). Overall, these findings may indicate some role for PSMA-targeted agents in providing a noninvasive imaging biomarker for the aggressiveness of disease, although similar findings in prostate cancer have so far not translated into a means to noninvasively risk-stratify patients.

Primary and metastatic colorectal adenocarcinoma has demonstrated uptake of the PSMA-targeted imaging agents ${ }^{111} \mathrm{In}-\mathrm{J} 591$ and ${ }^{68}$ Ga-PSMA-HBED-CC $(23,24,43-45)$. However, low uptake in rectal adenocarcinoma has been shown (46), and another group cautions about their experience with other non-PSMA-avid rectal cancers (43).

Again, several potential applications for PSMA-targeted agents are worthy of further exploration in well-designed trials. Similar to lung cancer, PSMA-targeted radiotracer uptake may be a surrogate for neovascular density and provide a means to predict response to neovasculature-targeted therapeutic agents. Furthermore, the use of neoadjuvant chemoradiotherapy can lead to significant posttreatment ${ }^{18} \mathrm{~F}-\mathrm{FDG}$ uptake that may be mistaken for residual tumor, and PSMA-targeted agents may have the advantage of less nonspecific inflammatory uptake in this context. As with any widely metastatic cancer, a possible role for PSMA-directed radiopharmaceutical therapy in advanced and progressive colorectal cancer can be considered.

\section{BLADDER CANCER}

Bladder cancer is the sixth most common type of cancer in the United States, with approximately 79,000 cases each year (14). The expression of PSMA on normal bladder transitional epithelium is severalfold lower than on normal prostatic tissue, which is already low enough to be unapparent on PSMA-targeted PET scans $(15,16,47)$. PSMA transcripts have been detected in transitional cell carcinoma (47). Although protein expression has been variable, the average expression in transitional cell carcinoma and tumor neovasculature has been relatively low, at $17 \%(0 \%-86 \%)$ and $29 \%(21 \%-100 \%)$, respectively $(8,10,11,15,19,48)$.

It is somewhat surprising, then, that multiple case reports of PSMA-targeted imaging in humans have shown activity in metastatic lesions $(23,24,49)$. However, more in keeping with the inconsistent protein expression seen with immunohistochemistry studies, in a series of 3 patients, only 1 patient with metastatic transitional cell carcinoma showed PSMA-targeted activity in lung and lymph node metastatic lesions, whereas primary and metastatic lesions in the other 2 patients did not show increased uptake (23).

Despite the questionable imaging potential (50), PSMA expression may have some prognostic value in transitional cell bladder cancer. PSMA-expressing transitional cell carcinoma has been associated with higher stage and significantly decreased patient survival (47). Although it is unlikely that PSMA-targeted imaging agents will play a significant role in the evaluation and staging of most patients with transitional cell carcinoma, the potential for PSMA-targeted radiotracer uptake to provide noninvasive prognostic information on tumor aggressiveness may warrant further evaluation with an eye toward offering PSMA-based therapeutics to a select subset of transitional cell carcinoma patients, particularly those with highly PSMA-expressing transitional cell carcinoma not responding to current standard-of-care chemotherapy or immunotherapy regimens.

\section{RENAL CELL CARCINOMA (RCC)}

Kidney cancer is the eighth most common type of malignancy in the United States, with an estimate of 64,000 new cases per year (14). Expression of PSMA is seen in the normal kidney, particularly in the proximal renal tubules at the level of the epithelium $(2,8,11,15)$. Interestingly, the expression in RCC tumor cells across all histologic types is low $(0 \%-5 \%)(2,8,11,16,51)$, suggesting that PSMA expression is lost during malignant transformation or that RCC may originate from non-PSMA-expressing cells (11). However, expression in the neovasculature of multiple types of RCC has been demonstrated (Table 2). For instance, expression of PSMA in clear cell RCC neovasculature has been seen in an average of $84 \%(75 \%-100 \%)$ of cases $(8,15,16,51-54)$. Non-clear cell subtypes of RCC have lower rates of PSMA expression: $61 \%$ (31\%-87\%) for chromophobe RCC and 28\% (10\%-73\%) for papillary RCC $(51,52,55)$.

PSMA expression is also seen in the neovasculature of approximately $58 \%(18 \%-93 \%)$ of oncocytomas (51-53), the most common benign primary renal mass. In combination with the intrinsic expression of PSMA in normal kidney parenchyma, this low PSMA expression indicates that PSMA-targeted imaging has no role in the evaluation of clinically localized renal masses. However, early results from studies examining the imaging of metastatic clear cell RCC with PSMA-targeted imaging agents have been promising (Fig. 2) (56). At least a dozen metastatic clear cell RCC cases have been published with a variety of PSMA-targeted PET radiotracers $\left({ }^{111} \mathrm{In}-\mathrm{J} 591,{ }^{68} \mathrm{Ga}-\mathrm{PSMA}-\mathrm{HBED}-\mathrm{CC}\right.$, and ${ }^{18} \mathrm{~F}-\mathrm{DCFPyL}$ [2-(3-(1carboxy-5-[(6- ${ }^{18} \mathrm{~F}$-fluoro-pyridine-3-carbonyl)-amino]-pentyl)-ureido)pentanedioic acid]). Uptake has been demonstrated in primary tumors, lymph nodes, and bone metastases, with reported SUVs ranging between 1.7 and 35 (23,57-63).

The high uptake of PSMA-targeted imaging agents in clear cell RCC metastatic lesions suggests the potential for PSMA-based radiopharmaceutical therapy. As with several other malignancies described in this review, the effect of targeting radiopharmaceutical therapy at endothelial cells instead of at epithelial tumor cells, such as with prostate cancer, is unknown. Given the proliferation of new therapeutic options for clear cell RCC, including multiple tyrosine kinase inhibitors and immune checkpoint inhibitors, the potential to combine PSMA-based radiopharmaceutical therapy with other systemic therapies seems promising.

Further, the use of neovasculature-targeting agents to treat metastatic clear cell RCC (in particular, the first-line tyrosine kinase inhibitors) is once more suggestive of the possibility that PSMA-based imaging may be used to predict response to therapy and to provide imaging evidence of early response to therapy. In those patients suspected of having oligometastatic clear cell RCC, the high sensitivity and specificity of PSMA-targeted imaging may allow for a more appropriate selection of patients to undergo focal therapy such as metastatectomy $(64,65)$.

\section{PRIMARY BRAIN TUMORS}

PSMA messenger RNA has been found in brain tissue $(47,66)$, with spliced versions of the PSMA protein found in the cytoplasm 


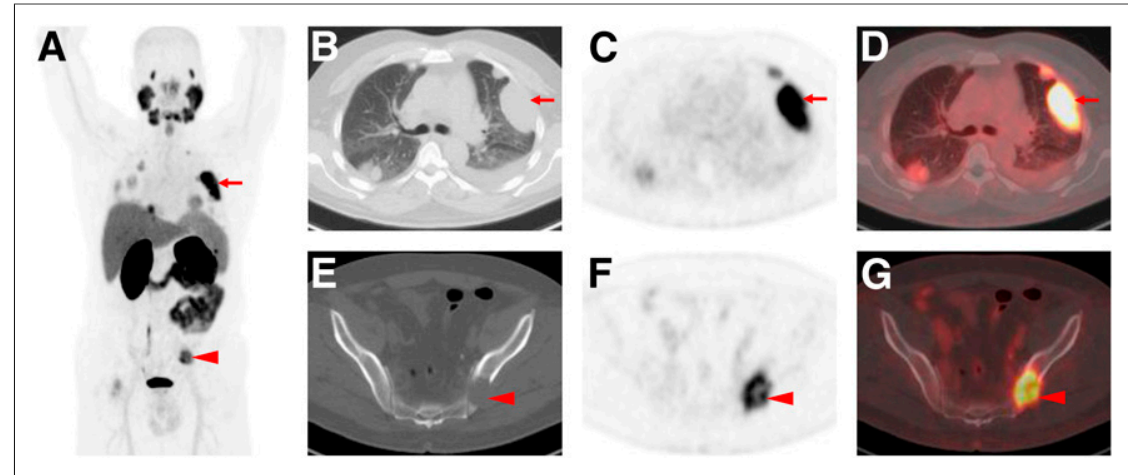

FIGURE 2. (A) Maximum-intensity-projection ${ }^{18} \mathrm{~F}$-DCFPyL PET image from patient with metastatic clear cell RCC is notable for multiple sites of varying degrees of abnormal radiotracer uptake, including lesions in lungs (arrow) and bones (arrowhead). (B-D) Axial unenhanced attenuation-corrected CT (B), axial ${ }^{18} \mathrm{~F}-\mathrm{DCFP}$ L PET (C), and axial ${ }^{18} \mathrm{~F}-\mathrm{DCFPyL}$ PET/CT (D) images through lungs show multiple nodules and masses with varying degrees of uptake, including dominant left-upperlobe mass with intense uptake (arrows). (E-G) Axial unenhanced attenuation-corrected CT (E), axial ${ }^{18} \mathrm{~F}$-DCFPyL PET (F), and axial ${ }^{18} \mathrm{~F}$-DCFPyL PET/CT (G) images through pelvis demonstrate lytic metastatic lesion in left posterior iliac bone with heterogeneous intense uptake (arrowheads).

$(15,16)$ and in the cell membrane (66). PSMA expression has been seen in astrocytes, hippocampal neurons, and the ependymal cell cytoplasm (16). PSMA binds to the $N$-methyl-D-aspartate receptor and a group II metabotropic glutamate receptor in the brain, releasing the neurotransmitter glutamate (66). PSMA expression is more pronounced and more localized to tumor neovasculature in highly vascular gliomas, such as glioblastoma multiforme, than in lower-grade gliomas $(21,67,68)$. These findings point toward a role for PSMA in neurotransmission and carcinogenesis (66).

Although the expression of PSMA in glioblastoma (World Health Organization grade IV) tumor cells is low (0\%-6\%) $(8,15,21,68)$, PSMA is consistently expressed in the tumor neovasculature $(8,21,67,68)$. Immunohistochemistry findings have been correlated in vivo with ${ }^{68} \mathrm{Ga}-\mathrm{PSMA}-\mathrm{HBED}-\mathrm{CC}$ and ${ }^{18} \mathrm{~F}-\mathrm{DCFPyL}$ PET/CT findings (68-70).

High-grade gliomas carry a poor prognosis, largely secondary to limited treatment options. Changes in treatment regimens favoring concurrent chemotherapy and radiation have improved survival (71). However, combination therapy can lead to radiation necrosis, pseudoprogression, and postradiation effects, all of which may show conventional-imaging findings similar to those of tumor progression (72). This imaging uncertainty occurs in approximately 1 of 5 patients receiving treatment (72) and can lead to additional brain biopsies or difficulties in treatment decisions. Absence of PSMAtracer uptake in normal brain parenchyma provides a favorable tumor-to-background ratio. PSMA-targeted imaging may potentially
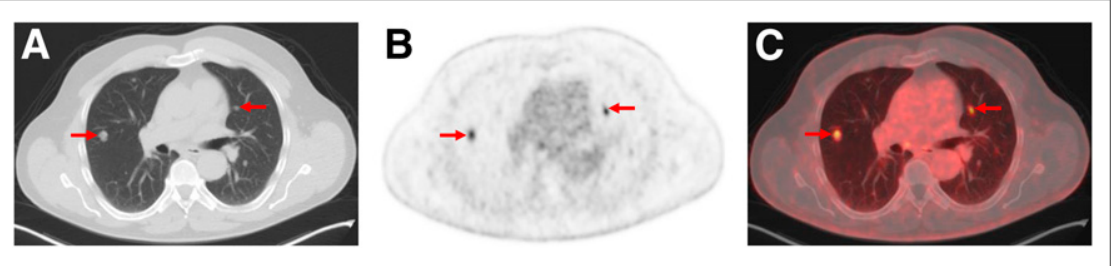

FIGURE 3. Axial unenhanced attenuation-corrected CT (A), axial ${ }^{18} \mathrm{~F}-\mathrm{DCFPyL} P E T(B)$, and axial ${ }^{18}$ F-DCFPyL PET/CT (C) images from 56-y-old man with known adenoid cystic carcinoma of salivary gland with widespread metastasis to lung. Intense radiotracer uptake is visible in multiple pulmonary nodules (arrows). therapies.

\section{OTHER CANCERS}

identify patients with disease recurrence and help direct changes in therapy. Patients with recurrence demonstrate a significantly higher $\mathrm{SUV}_{\text {max }}$ than patients without recurrence (70). Another potential application is the use of PSMA-targeted radiopharmaceuticals. Given the minimal uptake within normal brain parenchyma, radiopharmaceutical therapy specifically targeted to the neovasculature or tumor cells of high-grade gliomas could provide a new approach that is not fraught with the normal-tissue radiotoxicity that is often a result of external-beam radiation therapy.

\section{PANCREATIC CANCER}

Pancreatic cancer has an approximate incidence of 53,000 cases per year, with over half the patients having metastatic disease at the time of presentation (14), and 5-y survival is estimated at only $8 \%$ (14). Although immunohistochemistry has not demonstrated PSMA expression in normal pancreatic tissue $(2,8,15)$, pancreatic ductal adenocarcinoma tumor cells $(8,15)$ and tumor neovasculature (8) have been shown to have PSMA expression. Furthermore, patients with higher expression of PSMA in the pancreatic ductal adenocarcinoma neovasculature had significantly shorter survival (19). In vivo, ${ }^{111}$ In J591 (23) and ${ }^{68}$ Ga-PSMA-HBED-CC (73) imaging have demonstrated PSMA-targeted radiotracer uptake in both primary tumors and metastatic disease.

Adjuvant and neoadjuvant chemotherapy, radiation therapy, and tumor resection are treatment options that are deeply guided by imaging findings (74-78). Localizing a disease volume that is smaller than the threshold of current imaging modalities, and directing additional therapy to these areas, may lead to a much-needed improvement in patient survival and should be investigated in prospective studies. Furthermore, the potential for PSMA-targeted radiopharmaceutical therapy to be useful in treating pancreatic cancer should be studied, given the relative lack of effective systemic

PSMA expression has been seen in the neovasculature of multiple other types of solid tumor. For instance, neovasculature PSMA expression was seen in $76 \%$ of gastric adenocarcinoma samples, although no statistical association was found between tumor grade or survival and PSMA expression $(41,79)$. In oral squamous cell carcinoma, the tumor neovasculature of $75 \%$ of cases has demonstrated PSMA expression (79), with higher expression levels associated with decreased survival (79). Similarly, PSMA expression in osteosarcoma correlated with worse survival, the presence of pulmonary metastases, and larger tumor size, indicating its potential to be an independent osteosarcoma prognostic factor (80). Further, PSMAtargeted imaging is able to detect local recurrence and metastatic disease in adenoid cystic carcinoma (Fig. 3) (81), which expresses 
PSMA in ductal epithelial cells and the neovasculature. PSMA expression is also seen in the neovasculature of cervical cancer, endometrial cancer, and primary and metastatic ovarian cancer, with potential imaging and therapeutic implications (82).

\section{CONCLUSION}

The pathology literature reviewed herein has laid the groundwork for our understanding of the widespread expression of PSMA in numerous cancer types, particularly within tumor-associated neovasculature. For many of these cancers, the presence of PSMA provides the fields of molecular imaging and theranostics with numerous opportunities to undertake well-thought-out, prospective studies on the utility of PSMA-directed imaging or therapy to answer important clinical questions and to provide potential new therapies in difficult-to-treat disease phenotypes. Unfortunately, non-prostate cancer PSMA-based imaging is still predominantly limited to small case series and case reports, and positive incidental findings are much more likely to be reported in the literature than are negative incidental findings (i.e., tumors that lack PSMA-targeted radiotracer uptake). It is therefore quite likely that there is a significant selection bias in the currently published literature that limits our ability to know which nonprostate malignancies are most likely to benefit from PSMA-targeted imaging. This article is therefore a call to arms for imaging specialists and clinicians to answer the questions raised about PSMA-targeted imaging and therapy so we can understand the ultimate applicability of this new theranostic modality.

\section{DISCLOSURE}

Martin Pomper is a coinventor on a U.S. patent covering ${ }^{18} \mathrm{~F}$ DCFPyL and as such is entitled to a portion of any licensing fees and royalties generated by this technology. This arrangement has been reviewed and approved by the Johns Hopkins University in accordance with its conflict-of-interest policies. Michael Gorin has served as a consultant to Progenics Pharmaceuticals, the licensee of ${ }^{18}$ F-DCFPyL. Michael Gorin, Martin Pomper, and Steven Rowe have received research support from Progenics Pharmaceuticals. No other potential conflict of interest relevant to this article was reported.

\section{REFERENCES}

1. Horoszewicz JS, Kawinski E, Murphy GP. Monoclonal antibodies to a new antigenic marker in epithelial prostatic cells and serum of prostatic cancer patients. Anticancer Res. 1987;7:927-935.

2. Lopes AD, Davis WL, Rosenstraus MJ, Uveges AJ, Gilman SC. Immunohistochemical and pharmacokinetic characterization of the site-specific immunoconjugate CYT-356 derived from antiprostate monoclonal antibody 7E11-C5. Cancer Res. 1990;50:6423-6429.

3. Carter RE, Feldman AR, Coyle JT. Prostate-specific membrane antigen is a hydrolase with substrate and pharmacologic characteristics of a neuropeptidase. Proc Natl Acad Sci USA. 1996;93:749-753.

4. Pinto JT, Suffoletto BP, Berzin TM, et al. Prostate-specific membrane antigen: a novel folate hydrolase in human prostatic carcinoma cells. Clin Cancer Res. 1996;2:1445-1451.

5. Barrett AJ. Enzyme nomenclature: recommendations 1992—supplement 4: corrections and additions (1997). Eur J Biochem. 1997;250:1-6.

6. Baum RP, Kulkarni HR, Schuchardt C, et al. ${ }^{177}$ Lu-labeled prostate-specific membrane antigen radioligand therapy of metastatic castration-resistant prostate cancer: safety and efficacy. J Nucl Med. 2016;57:1006-1013.

7. Cantiello F, Gangemi V, Cascini GL, et al. Diagnostic accuracy of ${ }^{64}$ copper prostate-specific membrane antigen positron emission tomography/computer tomograph for primary lymph node staging of intermediate- to high-risk prostate cancer: our preliminary experience. Urology. 2017;106:139-145.
8. Chang SS, Reuter VE, Heston WD, Bander NH, Grauer LS, Gaudin PB. Five different anti-prostate-specific membrane antigen (PSMA) antibodies confirm PSMA expression in tumor-associated neovasculature. Cancer Res. 1999;59:3192-3198.

9. Liu H, Moy P, Kim S, et al. Monoclonal antibodies to the extracellular domain of prostate-specific membrane antigen also react with tumor vascular endothelium. Cancer Res. 1997;57:3629-3634.

10. Troyer JK, Beckett ML, Wright GL Jr. Detection and characterization of the prostate-specific membrane antigen (PSMA) in tissue extracts and body fluids. Int J Cancer. 1995;62:552-558.

11. Silver DA, Pellicer I, Fair WR, Heston WD, Cordon-Cardo C. Prostate-specific membrane antigen expression in normal and malignant human tissues. Clin Cancer Res. 1997;3:81-85.

12. Israeli RS, Powell CT, Corr JG, Fair WR, Heston WD. Expression of the prostate-specific membrane antigen. Cancer Res. 1994;54:1807-1811.

13. Kirchner J, Schaarschmidt BM, Sawicki LM, et al. Evaluation of practical interpretation hurdles in ${ }^{68} \mathrm{Ga}$-PSMA PET/CT in 55 patients. Clin Nucl Med. 2017; 42:e322-e327.

14. Howlader N, Noone AM, Krapcho M, et al, eds. SEER Cancer Statistics Review (CSR) 1975-2014. National Cancer Institute website. https://seer.cancer.gov/csr/ 1975_2014/. Published April 2017. Updated June 28, 2017. Accessed March 9, 2018.

15. Mhawech-Fauceglia P, Zhang S, Terracciano L, et al. Prostate-specific membrane antigen (PSMA) protein expression in normal and neoplastic tissues and its sensitivity and specificity in prostate adenocarcinoma: an immunohistochemical study using multiple tumour tissue microarray technique. Histopathology. 2007;50:472-483.

16. Kinoshita Y, Kuratsukuri K, Landas S, et al. Expression of prostate-specific membrane antigen in normal and malignant human tissues. World J Surg. 2006; 30:628-636.

17. Uría JA, Velasco G, Santamaría I, Ferrando A, López-Otín C. Prostate-specific membrane antigen in breast carcinoma. Lancet. 1997;349:1601.

18. Wernicke AG, Varma S, Greenwood EA, et al. Prostate-specific membrane antigen expression in tumor-associated vasculature of breast cancers. APMIS. 2014;122:482-489.

19. Ren H, Zhang H, Wang X, Liu J, Yuan Z, Hao J. Prostate-specific membrane antigen as a marker of pancreatic cancer cells. Med Oncol. 2014;31:857.

20. Sathekge M, Lengana T, Modiselle M, et al. ${ }^{68}$ Ga-PSMA-HBED-CC PET imaging in breast carcinoma patients. Eur J Nucl Med Mol Imaging. 2017;44:689-694.

21. Nomura N, Pastorino S, Jiang P, et al. Prostate specific membrane antigen (PSMA) expression in primary gliomas and breast cancer brain metastases. Cancer Cell Int. 2014;14:26.

22. Sasikumar A, Joy A, Nair BP, Pillai MRA, Madhavan J. False positive uptake in bilateral gynecomastia on ${ }^{68} \mathrm{Ga}$-PSMA PET/CT scan. Clin Nucl Med. 2017;42: e412-e414.

23. Milowsky MI, Nanus DM, Kostakoglu L, et al. Vascular targeted therapy with anti-prostate-specific membrane antigen monoclonal antibody J591 in advanced solid tumors. J Clin Oncol. 2007;25:540-547.

24. Pandit-Taskar N, O'Donoghue JA, Divgi CR, et al. Indium 111-labeled J591 antiPSMA antibody for vascular targeted imaging in progressive solid tumors. EJNMMI Res. 2015;5:28.

25. Sathekge M, Modiselle M, Vorster M, et al. ${ }^{68} \mathrm{Ga}$-PSMA imaging of metastatic breast cancer. Eur J Nucl Med Mol Imaging. 2015;42:1482-1483.

26. Medina-Ornelas SS, García-Pérez FO, Medel-Gamez C, Paredes-Amoroto E. A single brain metastasis seen on ${ }^{68} \mathrm{Ga}$-PSMA PET/CT in recurrent breast cancer. Rev Esp Med Nucl Imagen Mol. 2018;37:61-62.

27. Malik D, Basher RK, Mittal BR, Jain TK, Bal A, Singh SK. ${ }^{68}$ Ga-PSMA expression in pseudoangiomatous stromal hyperplasia of the breast. Clin Nucl Med. 2017;42:58-60.

28. NCCN clinical practice guidelines in oncology (NCCN Guidelines): breast cancer-version 4.2017. National Comprehensive Cancer Network website. https://www.nccn.org/professionals/physician_gls/pdf/breast.pdf. Published February 7, 2018. Accessed March 9, 2018.

29. Kulkarni HR, Singh A, Schuchardt C, et al. PSMA-based radioligand therapy for metastatic castration-resistant prostate cancer: the Bad Berka experience since 2013. J Nucl Med. 2016;57(suppl):97S-104S.

30. Wang HL, Wang S, Song W, et al. Expression of prostate-specific membrane antigen in lung cancer cells and tumor neovasculature endothelial cells and its clinical significance. PLoS One. 2015;10:e0125924.

31. Schmidt LH, Heitkötter B, Schulze AB, et al. Prostate specific membrane antigen (PSMA) expression in non-small cell lung cancer. PLoS One. 2017;12:e0186280.

32. Shetty D, Loh H, Bui C, Mansberg R, Stevanovic A. Elevated ${ }^{68} \mathrm{Ga}$ prostatespecific membrane antigen activity in metastatic non-small cell lung cancer. Clin Nucl Med. 2016;41:414-416.

33. Pyka T, Weirich G, Einspieler I, et al. ${ }^{68}$ Ga-PSMA-HBED-CC PET for differential diagnosis of suggestive lung lesions in patients with prostate cancer. $\mathrm{J} \mathrm{Nucl}$ Med. 2016;57:367-371. 
34. Shreve P, Faasse T. Role of positron emission tomography-computed tomography in pulmonary neoplasms. Radiol Clin North Am. 2013;51:767-779.

35. Meirelles G de SP, Capobianco J, de Oliveira MAC. Pitfalls and artifacts in the interpretation of oncologic PET/CT of the chest. Radiol Bras. 2017;50:55-59.

36. Ahuja A, Taneja S, Thorat K, Jena A. ${ }^{68}$ Ga-prostate-specific membrane antigenavid tubercular lesions mimicking prostate cancer metastasis on simultaneous prostate-specific membrane antigen PET/MRI. Clin Nucl Med. 2017;42:e509-e510.

37. Bouchelouche K, Vendelbo MH. Pulmonary opacities and bronchiectasis avid on ${ }^{68}$ Ga-PSMA PET. Clin Nucl Med. 2017;42:e216-e217.

38. Ahn JS, Ahn YC, Kim J-H, et al. Multinational randomized phase III trial with or without consolidation chemotherapy using docetaxel and cisplatin after concurrent chemoradiation in inoperable stage III non-small-cell lung cancer: KCSGLU05-04. J Clin Oncol. 2015;33:2660-2666.

39. Aupérin A, Le Péchoux C, Rolland E, et al. Meta-analysis of concomitant versus sequential radiochemotherapy in locally advanced non-small-cell lung cancer. J Clin Oncol. 2010;28:2181-2190.

40. Antonia SJ, Villegas A, Daniel D, et al. Durvalumab after chemoradiotherapy in stage III non-small-cell lung cancer. N Engl J Med. 2017;377:1919-1929.

41. Haffner MC, Kronberger IE, Ross JS, et al. Prostate-specific membrane antigen expression in the neovasculature of gastric and colorectal cancers. Hum Pathol. 2009;40:1754-1761.

42. Abdel-Hadi M, Ismail Y, Younis L. Prostate-specific membrane antigen (PSMA) immunoexpression in the neovasculature of colorectal carcinoma in Egyptian patients. Pathol Res Pract. 2014;210:759-763.

43. Huang Y-TT, Fong W, Thomas P. Rectal carcinoma on ${ }^{68} \mathrm{Ga}$-PSMA PET/CT. Clin Nucl Med. 2016;41:e167-e168.

44. Hangaard L, Jochumsen MR, Vendelbo MH, Bouchelouche K. Metastases from colorectal cancer avid on ${ }^{68}$ Ga-PSMA PET/CT. Clin Nucl Med. 2017;42:532-533.

45. Stoykow C, Huber-Schumacher S, Almanasreh N, Jilg C, Ruf J. Strong PSMA radioligand uptake by rectal carcinoma. Clin Nucl Med. 2017;42:225-226.

46. Sasikumar A, Joy A, Pillai MRA, Raman V, Vasudevan A, Madhavan J. A rare case of rectal carcinoma and prostate carcinoma with coexistent Paget's disease mimicking bone metastases in both ${ }^{18} \mathrm{~F}-\mathrm{FDG}$ and ${ }^{68} \mathrm{Ga}$ PSMA PET/CT. Eur $J$ Nucl Med Mol Imaging. 2017;44:173.

47. Gala JL, Loric S, Guiot Y, et al. Expression of prostate-specific membrane antigen in transitional cell carcinoma of the bladder: prognostic value? Clin Cancer Res. 2000;6:4049-4054.

48. Samplaski MK, Heston W, Elson P, Magi-Galluzzi C, Hansel DE. Folate hydrolase (prostate-specific membrane [corrected] antigen) 1 expression in bladder cancer subtypes and associated tumor neovasculature. Mod Pathol. 2011;24:1521-1529.

49. Gupta M, Choudhury P, Gupta G, Gandhi J. Metastasis in urothelial carcinoma mimicking prostate cancer metastasis in Ga-68 prostate-specific membrane antigen positron emission tomography-computed tomography in a case of synchronous malignancy. Indian J Nucl Med. 2016;31:222-224.

50. Campbell SP, Baras AS, Ball MW, et al. Low levels of PSMA expression limit the utility of ${ }^{18} \mathrm{~F}$-DCFPyL PET/CT for imaging urothelial carcinoma. Ann Nucl Med. 2018;32:69-74.

51. Spatz S, Tolkach Y, Jung K, et al. Comprehensive evaluation of prostate specific membrane antigen expression in the vasculature of renal tumors: implications for imaging studies and prognostic role. J Urol. 2018;199:370-377.

52. Baccala A, Sercia L, Li J, Heston W, Zhou M. Expression of prostate-specific membrane antigen in tumor-associated neovasculature of renal neoplasms. Urology. 2007;70:385-390.

53. Al-Ahmadie HA, Olgac S, Gregor PD, et al. Expression of prostate-specific membrane antigen in renal cortical tumors. Mod Pathol. 2008;21:727-732.

54. Chang SS, Reuter VE, Heston WD, Gaudin PB. Metastatic renal cell carcinoma neovasculature expresses prostate-specific membrane antigen. Urology. 2001;57: 801-805.

55. Gordon IO, Tretiakova MS, Noffsinger AE, Hart J, Reuter VE, Al-Ahmadie HA. Prostate-specific membrane antigen expression in regeneration and repair. Mod Pathol. 2008;21:1421-1427.

56. Rhee H, Blazak J, Tham CM, et al. Pilot study: use of gallium-68 PSMA PET for detection of metastatic lesions in patients with renal tumour. EJNMMI Res. 2016;6:76

57. Rowe SP, Gorin MA, Hammers HJ, Pomper MG, Allaf ME, Javadi MS. Detection of ${ }^{18} \mathrm{~F}$-FDG PET/CT occult lesions with ${ }^{18} \mathrm{~F}-\mathrm{DCFPyL} \mathrm{PET} / \mathrm{CT}$ in a patient with metastatic renal cell carcinoma. Clin Nucl Med. 2016;41:83-85.

58. Rowe SP, Deville C, Paller C, et al. Uptake of ${ }^{18} \mathrm{~F}$-DCFPyL in Paget's disease of bone, an important potential pitfall in clinical interpretation of PSMA PET studies. Tomography. 2015;1:81-84.

59. Sawicki LM, Buchbender C, Boos J, et al. Diagnostic potential of PET/CT using $\mathrm{a}^{68} \mathrm{Ga}$-labelled prostate-specific membrane antigen ligand in whole-body staging of renal cell carcinoma: initial experience. Eur J Nucl Med Mol Imaging. 2017; 44:102-107
60. Demirci E, Ocak M, Kabasakal L, et al. ${ }^{68} \mathrm{Ga}$-PSMA PET/CT imaging of metastatic clear cell renal cell carcinoma. Eur J Nucl Med Mol Imaging. 2014;41: 1461-1462.

61. Michaels EK, Blend M, Quintana JC. ${ }^{111}$ Indium-capromab pendetide unexpectedly localizes to renal cell carcinoma. J Urol. 1999;161:597-598.

62. Sasikumar A, Joy A, Nanabala R, Unni M, Tk P. Complimentary pattern of uptake in ${ }^{18} \mathrm{~F}$-FDG $\mathrm{PET} / \mathrm{CT}$ and ${ }^{68} \mathrm{Ga}$-prostate-specific membrane antigen PET/CT in a case of metastatic clear cell renal carcinoma. Clin Nucl Med. 2016; 41:e517-e519.

63. Siva S, Callahan J, Pryor D, Martin J, Lawrentschuk N, Hofman MS. Utility of ${ }^{68} \mathrm{Ga}$ prostate specific membrane antigen-positron emission tomography in diagnosis and response assessment of recurrent renal cell carcinoma. J Med Imaging Radiat Oncol. 2017;61:372-378.

64. Rowe SP, Gorin MA, Hammers HJ, et al. Imaging of metastatic clear cell renal cell carcinoma with PSMA-targeted ${ }^{18}$ F-DCFPyL PET/CT. Ann Nucl Med. 2015;29:877-882.

65. Gorin MA, Rowe SP, Hooper JE, et al. PSMA-targeted ${ }^{18}$ F-DCFPyL PET/CT imaging of clear cell renal cell carcinoma: results from a rapid autopsy. Eur Urol. 2017;71:145-146.

66. Luthi-Carter R, Barczak AK, Speno H, Coyle JT. Molecular characterization of human brain $\mathrm{N}$-acetylated alpha-linked acidic dipeptidase (NAALADase). J Pharmacol Exp Ther. 1998;286:1020-1025.

67. Wernicke AG, Edgar MA, Lavi E, et al. Prostate-specific membrane antigen as a potential novel vascular target for treatment of glioblastoma multiforme. Arch Pathol Lab Med. 2011;135:1486-1489.

68. Salas Fragomeni RA, Menke JR, Holdhoff M, et al. Prostate-specific membrane antigen-targeted imaging with $\left[{ }^{18} \mathrm{~F}\right] \mathrm{DCFPyL}$ in high-grade gliomas. Clin Nucl Med. 2017;10:e433-e435.

69. Schwenck J, Tabatabai G, Skardelly M, et al. In vivo visualization of prostatespecific membrane antigen in glioblastoma. Eur J Nucl Med Mol Imaging. 2015;42:170-171.

70. Sasikumar A, Joy A, Pillai MRA, et al. Diagnostic value of ${ }^{68}$ Ga PSMA-11 PET/ CT imaging of brain tumors: preliminary analysis. Clin Nucl Med. 2017;42:e41e48.

71. Stupp R, Mason WP, van den Bent MJ, et al. Radiotherapy plus concomitant and adjuvant temozolomide for glioblastoma. N Engl J Med. 2005;352:987-996.

72. Kruser TJ, Mehta MP, Robins HI. Pseudoprogression after glioma therapy: a comprehensive review. Expert Rev Neurother. 2013;13:389-403.

73. Sahbai S, Rieping $P$, Pfannenberg C, la Fougère $C$, Reimold M. Pancreatic ductal adenocarcinoma with high radiotracer uptake in ${ }^{68} \mathrm{Ga}$-prostate-specific membrane antigen PET/CT. Clin Nucl Med. 2017;9:717-718.

74. Gudjonsson B, Livstone EM, Spiro HM. Cancer of the pancreas: diagnostic accuracy and survival statistics. Cancer. 1978;42:2494-2506.

75. Neoptolemos JP, Stocken DD, Friess H, et al. A randomized trial of chemoradiotherapy and chemotherapy after resection of pancreatic cancer. $N$ Engl $J$ Med. 2004;350:1200-1210.

76. Gudjonsson B. Carcinoma of the pancreas: critical analysis of costs, results of resections, and the need for standardized reporting. J Am Coll Surg. 1995;181: 483-503.

77. Sugarbaker PH. Strategies to improve local control of resected pancreas adenocarcinoma. Surg Oncol. 2017;26:63-70.

78. Winter JM, Cameron J, Campbell K, et al. 1423 pancreaticoduodenectomies for pancreatic cancer: a single-institution experience. J Gastrointest Surg. 2006;10; 1199-1210.

79. Haffner MC, Laimer J, Chaux A, et al. High expression of prostate-specific membrane antigen in the tumor-associated neo-vasculature is associated with worse prognosis in squamous cell carcinoma of the oral cavity. Mod Pathol. 2012;25:1079-1085.

80. Zeng C, Ke Z-F, Yang Z, et al. Prostate-specific membrane antigen: a new potential prognostic marker of osteosarcoma. Med Oncol. 2012;29:2234-2239.

81. Klein Nulent TJW, van Es RJJ, Krijger GC, de Bree R, Willems SM, de Keizer B. Prostate-specific membrane antigen PET imaging and immunohistochemistry in adenoid cystic carcinoma: a preliminary analysis. Eur J Nucl Med Mol Imaging. 2017;44:1614-1621.

82. Wernicke AG, Kim S, Liu H, Bander NH, Pirog EC. Prostate-specific membrane antigen (PSMA) expression in the neovasculature of gynecologic malignancies: implications for PSMA-targeted therapy. Appl Immunohistochem Mol Morphol. February 9, 2016 [Epub ahead of print].

83. Rhee $\mathrm{H}, \mathrm{Ng} \mathrm{KL}$, Tse BW-C, et al. Using prostate specific membrane antigen (PSMA) expression in clear cell renal cell carcinoma for imaging advanced disease. Pathology. 2016;48:613-616.

84. Rowe SP, Gage KL, Faraj SF, et al. ${ }^{18}$ F-DCFBC PET/CT for PSMA-based detection and characterization of primary prostate cancer. J Nucl Med. 2015;56: $1003-1010$ 